\title{
Juvenile Root Traits Show Limited Correlation with Grain Yield, Yield Components and Grain Mineral Composition Traits in Indian Wheat under Hostile Soils
}

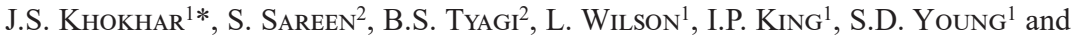 \\ M.R. BROADLEY ${ }^{1}$
}

${ }^{1}$ Plant and Crop Sciences, University of Nottingham, Loughborough, United Kingdom

${ }^{2}$ Indian Institute of Wheat \& Barley Research, Karnal, Haryana, India

(Received 16 November 2018; Accepted 5 February 2019;

Communicated by M. Taylor)

\begin{abstract}
Correlations between juvenile wheat root traits, and grain yield and yield component traits under optimal field conditions have previously been reported in some conditions. The aim of this study was to test the hypothesis that juvenile wheat root traits correlate with yield, yield components and grain mineral composition traits under a range of soil environments in India. A diverse panel of 36 Indian wheat genotypes were grown for ten days in 'pouch and wick' high-throughput phenotyping (HTP) system (20 replicates). Correlations between juvenile root architecture traits, including primary and lateral root length, and grain yield, yield components and grain mineral composition traits were determined, using field data from previously published experiments at six sites in India. Only a limited number of juvenile root traits correlated with grain yield (GYD), yield components, and grain mineral composition traits. A narrow root angle, potentially representing a 'steep' phenotype, was associated with increased GYD and harvest index (HI) averaged across sites and years. Length related root traits were not correlated with GYD or HI at most sites, however, the total length of lateral roots and lateral root number correlated with GYD at a sodic site of $\mathrm{pH}$ 9.5. The total length of lateral roots (TLLR) correlated with grain zinc ( $\mathrm{Zn}$ ) concentration at one site. A wider root angle, representing a shallow root system, correlated with grain iron $(\mathrm{Fe})$ concentration at most sites. The total length of all roots (TLAR) and total length of primary roots (TLPR) correlated with grain $\mathrm{S}$ concentration at most sites. Narrow root angle in juvenile plants could be a useful proxy trait for screening germplasm for improved grain yield. Lateral root and shallow root traits could potentially be used to improve grain mineral concentrations. The use of juvenile root traits should be explored further in wheat breeding for diverse environments.
\end{abstract}

Keywords: wheat, root traits, nutrients, hostile acidic soils, grain yield and components

*Corresponding author; E-mail: jaswant.singh1@nottingham.ac.uk (JSK)

Open Access. This is an open-access article distributed under the terms of the Creative Commons AttributionNonCommercial 4.0 International License (https://creativecommons.org/licenses/by-nc/4.0/), which permits unrestricted use, distribution, and reproduction in any medium for non-commercial purposes, provided the original author and source are credited, a link to the CC License is provided, and changes - if any - are indicated. 


\section{Introduction}

Root architecture describes the spatial configuration of plant roots in soil, which is important for anchorage, nutrient/water uptake, and for responding to biotic and abiotic stresses (Lynch 2007; Gewin 2010; De Smet 2012). A 'steep, cheap and deep' root system could make plants more efficient in water and nutrient uptake from deeper layers of soil, even under less fertile and drought conditions (Lynch 2013). Whilst the Green Revolution increased the grain production tremendously based on selection of shoot traits, including semi-dwarf varieties (Khush 2001), modern wheat varieties have root biomass less than two-third of the mean of wheat landraces (Waines and Ehdai 2007). Root traits have not yet been selected directly in wheat breeding to our knowledge.

Wheat has a dense root system which is influenced by both genetic and environmental factors. Their root system consists of three to eight primary roots (also called seminal roots). The secondary root system, comprising adventitious/nodal roots, arises from basal nodes of the coleoptile and stem; adventitious root length, thickness and numbers increase with numbers of leaves and tillers (Hoad et al. 2001; Bradley et al. 2008). There is genotypic variation for root length density (RLD) in wheat germplasm in different soil horizons. For example, an average RLD in top soil $(0-20 \mathrm{~cm})$ was $7 \mathrm{~cm} \mathrm{~cm}^{-3}$ and in lower soil profile (80-100 cm depth) was $0.2 \mathrm{~cm} \mathrm{~cm}^{-3}$ in a winter wheat measured at anthesis in field experiments, and soil environmental conditions such as soil $\mathrm{pH}$, soil texture, moisture level and other environmental stresses affected the root spatial configuration in soil (Pillinger et al. 2005).

Studying wheat root traits in field condition is challenging, therefore researchers have started to deploy high throughput phenotyping (HTP) systems to screen seedling root traits. For example, Bai et al. (2013) used a germination paper based 'cigar roll' system to grow 199 double haploid wheat near isogenic lines (NILs) for 11 days. They reported no correlation of primary root length and lateral root length with plant height at maturity in the field. Among 52 wheat genotypes, McDonald (2010) reported that varieties with a narrow root angle between pairs of primary seminal roots of 14 days old seedlings grown in root boxes showed slightly greater grain yield than wider root angle varieties in most of the breeding field trials.

A 'pouch \& wick' germination paper based approach was used to grow 94 double haploid wheat lines in nutrient solution for nine days to study root architectural traits by Atkinson et al. (2015). They reported no correlation between seedling root traits and grain yield and plant height, measured in field experiments with high and low nitrogen treatments. Xie et al. (2017) grew 226 recombinant inbred lines (RIL) in 'pouch \& wick' growth system to study the root traits of 13 days old seedlings and to test the association of seedling root traits with grain yield and yield components of inbred wheat lines measured under field conditions. They reported that the maximum width and depth of roots correlated with grain yield harvested in field, and maximum width root traits also associated with grain $\mathrm{m}^{-2}$ and spikes $\mathrm{m}^{-2}$ measured in field trials. The total root length and primary root number of 13 days old seedlings correlated positively with numbers of grain per spike and biomass per unit area under field conditions. There were negative relation- 
ships of total root length and primary root number with 1000 grain weight of 226 RILs measured in field conditions. In contrast, total root length and primary root number showed negative correlation with 1000 grain weight measured in glasshouse pot experiment. However, they did not show any relationships between root emergence angles of primary roots with grain yield in the field. Clearly, the association of juvenile root architecture traits with grain yield and yield components in the field warrants further exploration.

In this study, we used this same germination paper based HTP 'pouch \& wick' system to characterise the juvenile root architectural traits of a diverse panel of 36 widely adapted Indian genotypes. The aim of this study was to test the hypothesis that juvenile root traits correlate with yield, yield components and grain mineral composition traits measured under a range of soil environmental conditions in India, including hostile soil conditions. Data of grain yield and component traits, and mineral composition traits of 36 genotypes grown at six different environmental sites ( $\mathrm{pH} 4.5$ to 9.5) in India in 2013-2014 and 20142015 have been used from Khokhar et al. (2017) and Khokhar et al. (2018).

\section{Materials and Methods}

\section{Wheat genotypes, field experiments and grain mineral analysis}

A panel of 36 elite wheat genotypes comprising Triticum aestivum L. $(\mathrm{n}=34)$ and Triticum durum L. $(\mathrm{n}=2)$ were grown at six sites in India in 2013-2014 and 2014-2015. The six sites were, (1) Indian Institute of Wheat and Barley Research (IIWBR), Karnal, Haryana (HR); (2) IIWBR, Hisar (HR); (3) Narendra Deva University of Agriculture and Technology (NDUA\&T), Kumarganj, Faizabad, Uttar Pradesh (UP), reclaimed site; (4) NDUA\&T, Kumarganj, Faizabad (UP), sodic site; (5) Uttar Banga Krishi Viswavidyalaya (UBKV), Malda (WB) and (6) UBKV, Pundibari, Cooch Behar, West Bengal (WB). All genotypes and experimental conditions are described in Khokhar et al. (2017), including data for yield and yield components. Grain mineral composition traits were measured from samples collected from five sites, except Pundibari, in 2013-2014 and from all six sites in 2014-2015, as reported in Khokhar et al. (2018).

\section{High throughput root phenotyping platform and procedure}

\section{The HTP platform}

A two dimensional (2D) hydroponic-based 'pouch and wick' HTP platform was used to study juvenile root architectural traits of Indian wheat genotypes under controlled conditions. The HTP system is described by Atkinson et al. (2015), Thomas et al. (2016a), and Xie et al. (2017). In brief, the system comprised a set of aluminium frames $(104 \times 62 \times 102$ $\mathrm{cm}$; KJN Ltd., Leicester, UK). Each frame had two long black polypropylene sheets $(101 \times 31 \times 0.3 \mathrm{~cm})$ and two short black polypropylene sheets $(63 \times 31 \times 0.3 \mathrm{~cm})$ covering the frame from all sides to maintain darkness within the frame (Fig. 1B). In this system, each pouch comprised blue germination paper $(24 \times 30 \mathrm{~cm}$, SD 7640; Anchor paper 


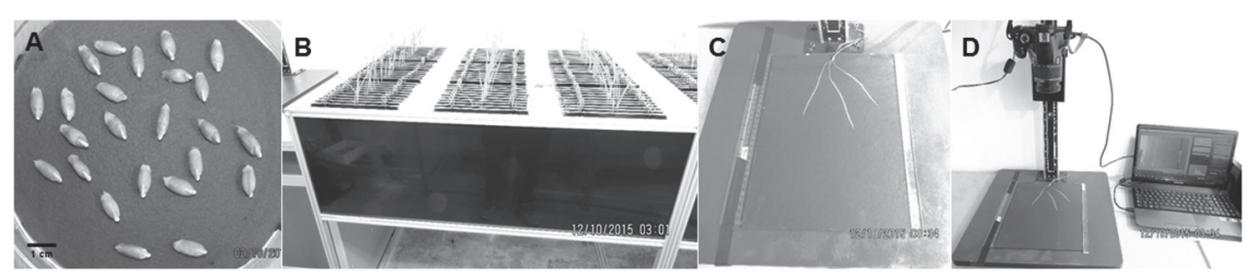

Figure 1. (A) Grain were soaked on germination paper (B) Wheat seedlings growing in hydroponic growth frame (C) Juvenile root image after 10 days of sowing (D) Copy stand with mounted camera above germination paper with juvenile root for imaging

Company, St. Paul, MN, USA), covered with black polythene film of the same size, and attached to an acrylic rod $(316 \times 15 \times 5 \mathrm{~mm}$; Acrylic Online Hull, UK) using two fold back clips. The growth pouches with acrylic rod were suspended above drip trays using a toothed acrylic holder fixed to the aluminium frame. Each aluminium frame comprised 9 drip trays arranged in 3 columns and 3 rows. The trays contained 10 or 11 pouches, with 96 pouches per frame. Each drip tray contained $2 \mathrm{~L}$ of $50 \%$ strength Hoagland's solution (No. 2 Basal salt Mixture, Sigma Aldrich, Dorset, UK) prepared with deionised water. Every three days during an experiment, $500 \mathrm{~mL}$ deionised water was added to the drip trays to ensure germinated paper remained saturated.

Grain of the 36 genotypes were sieved using a set of calibrated sieves (Scientific Laboratory Supplies Ltd., Hessle, UK) and grain of size within a range 3.35-4 mm were used. Grains were sterilized by $5 \%(\mathrm{v} / \mathrm{v})$ sodium hypochloride for 15 minutes and then rinsed three times with sterilized water. Grains were placed on pre-soaked germination paper, crease side down and incubated at $4{ }^{\circ} \mathrm{C}$ for 24 hours (Fig. 1A). Subsequently, grains were sown onto the pouches by placing a single seed ventrally (side has the deep crease), with embryo pointed downwards, $\sim 2 \mathrm{~cm}$ from the top edge on the germinated paper; the black polythene sheet held the grain in place. Two grains were grown per pouch, one on each side. Thus, a total of 192 plants were grown per frame. Two frames were used in each experiment run, to give a potential size of 384 plants per run. Two runs were carried out to get the 20 replicates of each genotype. Atkinson et al. (2015) recommended at least 15 replicates to detect significant differences in juvenile root architecture traits in wheat. The frames were placed in controlled environment room $\left(12 \mathrm{~h}\right.$ photoperiod: $20^{\circ} \mathrm{C}$ day, $15{ }^{\circ} \mathrm{C}$ night, with a light intensity of $400 \mu \mathrm{mol} \mathrm{m}^{-2} \mathrm{~s}^{-1}$ Photosynthetically Active Radiation (PAR).

\section{Root imaging and analysis}

Ten days after sowing (DAS), pouches were transferred to a copy stand, the black polythene sheets were removed from each pouch carefully (Fig. 1C) and juvenile root images were taken using a Digital Single Lens Reflex (DSLR) camera (Canon EOS 1100D, Canon Inc., Tokyo, Japan) fitted on copy stand (model SGCS-920; Speed Graphic, Hampshire, UK) at specific height, using canon software (Fig. 1D). 
The root images were renamed with their corresponding genotypic names in experiment. All images were cropped using ImageJ software (https://imagej.nih.gov/ij/). Cropped root images were processed and analysed to quantify the different juvenile root traits (Table S1*; Figure S1) using RootNav software (Pound et al. 2013). The juvenile root traits measured are described in Table S1.

\section{Statistical analysis}

Pearson correlation coefficients were used to determine if mean juvenile root traits in HTP correlated with field traits, and also among the juvenile root traits themselves. Variance components were calculated to determine the contribution of genotype to variation in the juvenile root traits. The power of the experiment to detect differences in juvenile root trait means between genotypes, as a function of the number of replicates, was determined and visualised as contour plots (c.f. Thomas et al. 2016a). All data analyses were conducted using GenStat $17^{\text {th }}$ Edition (VSN International Ltd., Hemel Hempstead, UK).

\section{Results}

A panel of the 36 Indian wheat genotypes varied from 4 to 6 in primary root numbers (PRN), 37 to $67 \mathrm{~cm}$ in total length of all roots (TLAR), 0.1 to $0.9 \mathrm{~cm}$ in total length of lateral roots (TLLR) and, from 49 to 96 degree in emergence angle between the outermost pair of primary roots measured at 25\% length of root (PEA 251), when grown on germinated paper in HTP system for 10 days, averaged across $\sim 20$ replicates.

\section{Relationships of length related juvenile root traits with grain yield and yield components}

The length-related root traits (CHCRY, CHCRX, CMRX, CMRY, MDR, TLAR, TLPR, $\mathrm{RCH})$ did not show significant correlations with mean grain yield (GYD, $\mathrm{t} \mathrm{ha}^{-}$), biological yield (BYD, g), harvest index (HI \%), 1000 grain weight (TGW, g) and plant height (PHT, cm), of 36 genotypes averaged across sites and years (Table S2). The maximum width of root (MWR) and total length of lateral roots (TLLR) showed positive relationships with PHT of 36 genotypes, averaged across sites and years $(r=0.42$ and 0.52 , respectively) (Fig. 2).

The total length of lateral roots (TLLR) correlated with GYD $(r=0.43)$ and BYD $(r=0.47)$ of 36 genotypes, averaged across years, only at Kumarganj-sodic site. The TLLR showed positive correlation with PHT of 36 genotypes, averaged across years, at all sites $(\mathrm{P}<0.05)$ except at Kumarganj-sodic and Pundibari (acidic soil) sites.

The mean GYD, TGW and HI of 36 genotypes averaged across years, did not show significant correlations with any juvenile root length traits at Karnal, Hisar (saline), Kumarganj-reclaimed (saline), Malda and Pundibari (acidic) sites, except Kumarganjsodic site.

*Further details about the Electronic Supplementary Material (ESM) can be found at the end of the article. 


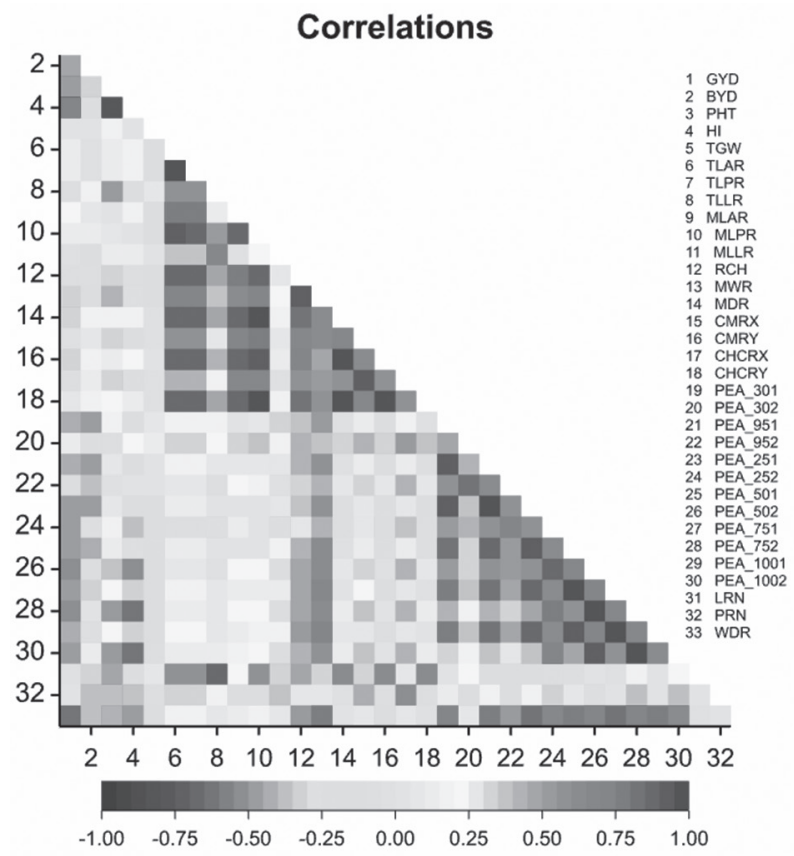

Figure 2. Correlation coefficients (r) between juvenile root traits measured in HTP and grain yield and component traits of 36 wheat genotypes grown at six sites over two years (2013-2014 and 2004-2015). Colour represents strength of correlation from strongly negative (dark blue) to strongly positive (dark red). Abbreviations are in Table S1

\section{Relationships of juvenile root angle traits with grain yield and yield components}

A narrow root angle between primary roots showed a positive correlation with GYD and component traits of 36 genotypes, averaged across sites and years. For example, emergence angle between the outermost pair of primary roots measured at $25 \%$ length of root (PEA 251) and emergence angle between the outermost pair of primary roots measured at 30 pixel (PEA 301) correlated negatively with GYD of 36 genotypes, averaged across sites and years $(r=-0.46$ and $-0.44 ; \mathrm{P}<0.05$, respectively). Similarly, BYD of 36 genotypes, averaged across sites and years, showed negative relationships with PEA 251 and PEA 301, ( $\mathrm{r}=-0.49,-0.48 ; \mathrm{P}<0.05$, respectively) (Fig. 2).

A narrow juvenile root angle traits showed weak positive relationships with GYD of 36 genotypes averaged across years, at Karnal (Table S3), Kumarganj-reclaimed (Table S5), Malda (Table S7) and Pundibari (Table S8) sites $(\mathrm{P}<0.01)$. For example, emergence angle between the second inner pair of primary roots, measured at $75 \%$ length of root (PEA 752), correlated negatively with GYD of 36 genotypes, averaged across years, at Karnal ( $\mathrm{r}=-0.42)$ (Fig. 3A), Kumarganj-reclaimed $(\mathrm{r}=-0.44)$ (Fig. 3B), Malda ( $\mathrm{r}=-0.44)$ (Fig. 3C) and Pundibari (acidic soil) ( $\mathrm{r}=-0.51)$ (Fig. 3D) sites. The PEA 752 trait correlated negatively with $\mathrm{HI}$ of 36 genotypes, averaged across years, at Karnal $(\mathrm{r}=-0.60)$, Hisar $(\mathrm{r}=-0.69)$, Kumarganj-reclaimed $(\mathrm{r}=-0.69)$ and Malda $(\mathrm{r}=-0.53)$ sites. 


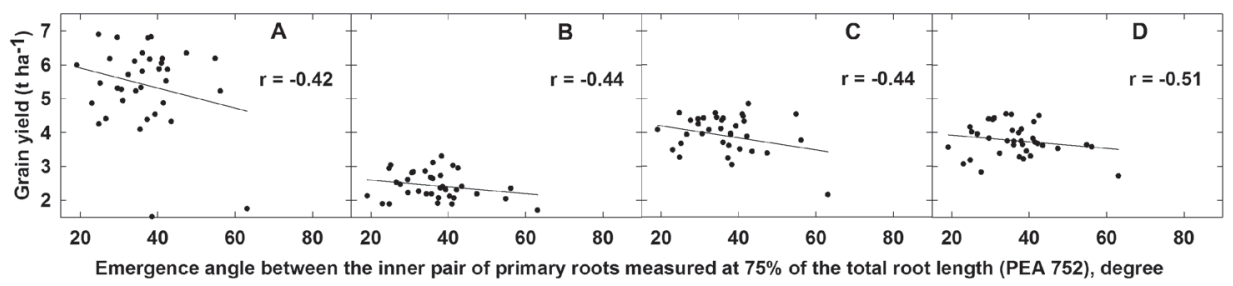

Figure 3. Correlations between grain yields of 36 wheat genotypes measured in field and juvenile root angle trait, PEA 752, measured in HTP system. Grain yield data are the mean of 36 genotypes with two replicates over two years, 2013-2014 and 2014-2015 at Karnal (A), Kumarganj-reclaimed (B), Malda (C) and Pundibari

(D). Root angle data are the mean of 36 genotypes with 20 replicates

A wider root angle was weakly positively correlated with PHT of 36 genotypes, averaged across years, at all the sites except at Pundibari $(\mathrm{P}<0.05)$. For example, PEA 752 trait correlated positively with PHT of 36 genotypes, averaged across years, at Karnal $(\mathrm{r}=0.49)$, Hisar $(\mathrm{r}=0.54)$, Kumarganj-reclaimed $(\mathrm{r}=0.42)$, Kumarganj-sodic $(\mathrm{r}=0.41)$ and Malda $(\mathrm{r}=0.53)$ sites. The emergence angle between the inner pair of primary roots measured at 30 pixel $(0.8 \mathrm{~cm})$ length from the seed (PEA 302) correlated positively with TGW of 36 genotypes, averaged across years at Hisar (saline soil) site $(r=0.46, \mathrm{P}<0.05)$.

\section{Correlations between length-related juvenile root traits, grain Zn concentration, and the grain ionome}

Grain Zn concentration has previously been reported for these 36 genotypes (Khokhar et al. 2018) because this micronutrient is an important breeding target for grain quality and human nutrition. In the field, grain $\mathrm{Zn}$ concentration of 36 wheat genotypes ranged from 25 to $35 \mathrm{mg} \mathrm{kg}^{-1}$. There was little evidence of association between length-related juvenile root traits and grain $\mathrm{Zn}$ concentration. Grain $\mathrm{Zn}$ concentration averaged across years, correlated with the TLLR only at Pundibari (acidic) site $(\mathrm{r}=0.45 ; \mathrm{P}<0.05)$.

Among the macronutrients, grain magnesium $(\mathrm{Mg})$ concentration of the 36 genotypes, averaged across years, correlated with LRN $(r=0.43)$ and TLLR $(r=0.58)$ at Karnal, TLLR $(r=0.47)$ at Hisar, MWR $(r=0.48)$ at Kumarganj-reclaimed site, LRN $(r=0.53)$ at Malda and with LRN $(\mathrm{r}=0.47)$ and TLLR $(\mathrm{r}=0.44)$ at Pundibari site. The grain sulphur (S) concentration of the 36 genotypes, averaged across years, correlated with most of the length related juvenile roots traits (TLAR, TLPR, TLLR, MLLR, MWR, CHCRY) and other juvenile root traits (LRN, PRN and RCH) at most of the sites, except at Kumarganj-sodic site ( $\mathrm{r}$ between 0.42 and 0.65 ; P between $<0.05$ and $<0.001$ ). For example, mean grain sulphur $\mathrm{S}$ concentration of the 36 genotypes, averaged across years, correlated with total length of all roots (TLAR) and total length of primary roots (TLPR) at Karnal $(r=0.47 ; r=0.46$, respectively), Hisar $(r=0.51 ; r=0.50$, respectively), Kumarganjreclaimed $(\mathrm{r}=0.63 ; \mathrm{r}=0.63$, respectively), Malda $(\mathrm{r}=0.44 ; \mathrm{r}=0.43$, respectively $)$ and Pundibari ( $\mathrm{r}=0.55 ; \mathrm{r}=0.55$, respectively) sites (Tables S3, S4, S5, S7 and S8, respectively). 
Among the micronutrients other than $\mathrm{Zn}$, grain copper $(\mathrm{Cu})$ concentration of the 36 genotypes, averaged across years, correlated positively with MWR $(r=0.43)$ at Hisar and negatively with primary root number (PRN; $r=-0.45)$ at Pundibari site. The mean grain Fe concentration of the 36 genotypes, averaged across sites and years, correlated with MWR $(r=0.63)$, root convex hull area $(\mathrm{RCH} ; \mathrm{r}=0.48)$ and TLLR $(\mathrm{r}=0.42)$. However, grain Fe concentration of the 36 genotypes, averaged across years, correlated with MWR at Karnal $(\mathrm{r}=0.58)$, Hisar $(\mathrm{r}=0.48)$ and at Malda $(\mathrm{r}=0.55)$ sites. The grain manganese $(\mathrm{Mn})$ concentration of the 36 genotypes, averaged across years, correlated with LRN at Karnal $(r=0.43)$ and Malda $(r=0.55)$ and with TLLR at Kumarganj-reclaimed $(r=0.46)$ site. Among the non-essential elements, the grain arsenic (As) concentration of the 36 genotypes, averaged across years, correlated with TLAR and TLPR $(r=0.42)$ only at Karnal site. The grain selenium (Se) concentration of 36 genotypes, averaged across years, correlated with PRN at Karnal $(\mathrm{r}=0.44)$, maximum width of roots $(\mathrm{r}=0.42)$ and root convex hull area $(\mathrm{RCH})$ at Kumarganj-reclaimed $(\mathrm{r}=0.43)$ site and with LRN at Malda $(r=0.49)$ site.

\section{Correlations between angle-related juvenile root traits, grain Zn concentration and the grain ionome}

Grain $\mathrm{Zn}$ concentration of 36 genotypes, averaged across years, did not correlate with root angle traits at any site.

Among the macronutrients, grain calcium $(\mathrm{Ca})$ concentration of the 36 genotypes, averaged across years, correlated positively with PEA $302(\mathrm{r}=0.53)$ at Hisar, PEA 252 $(\mathrm{r}=0.42)$ at Kumarganj-reclaimed and with PEA $251(\mathrm{r}=0.54)$ at Pundibari sites. The grain potassium $(\mathrm{K})$ concentration of the 36 genotypes, averaged across years, correlated positively with wider root angle traits (PEA 251, PEA 301, PEA 501, PEA 751, PEA 951) at Karnal site ( $\mathrm{r}$ between 0.43 and $0.58 ; \mathrm{P}<0.05$ ). The grain $\mathrm{Mg}$ concentration of the 36 genotypes, averaged across years, correlated positively with PEA $301(\mathrm{r}=0.51, \mathrm{P}<0.05)$ only at Karnal site. The grain $\mathrm{S}$ concentration of 36 genotypes, averaged across years, correlated negatively with emergence angle between the second inner pair of primary roots measured at 25\% length of root (PEA 252; $\mathrm{r}=-0.48, \mathrm{P}<0.05)$, emergence angle between the second inner pair of primary roots measured at $50 \%$ length of root (PEA 502; $\mathrm{r}=-0.49, \mathrm{P}<0.05)$ and with PEA $752(\mathrm{r}=-0.53, \mathrm{P}<0.01)$ juvenile root angle traits at Pundibari (acidic soil) site.

Among the micronutrients, the grain $\mathrm{Cu}$ concentration of the 36 genotypes, averaged across years, correlated with wider root angle traits which potentially represent the shallow root system, at Malda site. For example, the grain $\mathrm{Cu}$ concentration of the 36 genotypes, averaged across years, correlated positively with PEA $302(\mathrm{r}=0.46, \mathrm{P}<0.05)$ at Malda site. The grain Fe concentration of the 36 genotypes, averaged across years, correlated positively with PEA 251 at Karnal (Fig. 4A), at Kumarganj-reclaimed (Fig. 4B) and at Malda (Fig. 4C) sites. The grain molybdenum (Mo) concentration of the 36 genotypes, averaged across years, correlated with narrow root angle traits which potentially represent the steep root angle system, at Hisar and Kumarganj-sodic (Table S6) sites. For 


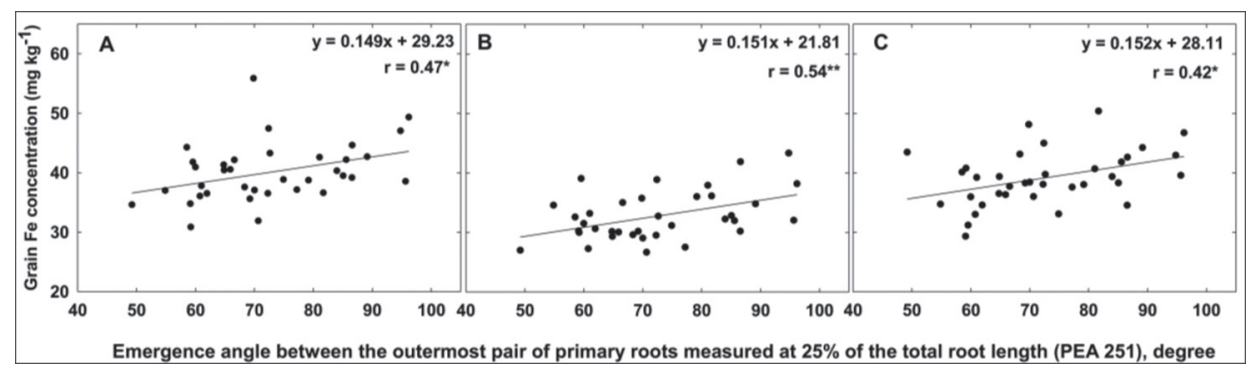

Figure 4. Correlation of mean grain Fe concentration of 36 genotypes grown at Karnal (A), Kumarganjreclaimed (B) and Malda (C) sites with juvenile root angle trait, PEA 251 measured in HTP system. Grain Fe concentration data are the mean of two replicate per plot of 36 genotypes over two years (2013-2014 and 2014-2015) at Karnal and Malda sites and one replicate per plot in 2013-2014 and two replicate per plot in 2014-2015 at Kumarganj-reclaimed site. $* \mathrm{P}<0.05 ; * * \mathrm{P}<0.01$

example, grain Mo concentration of the 36 genotypes, averaged across years, correlated negatively with PEA $502(\mathrm{r}=-0.49, \mathrm{P}<0.05)$ at Hisar and with PEA $251(\mathrm{r}=-0.51$, $\mathrm{P}<0.05)$ at Kumarganj-sodic site. Among the non-essential elements, grain As concentration of the 36 genotypes correlated negatively with PEA 502 and PEA $752(\mathrm{r}=-0.43$, $\mathrm{P}<0.05)$ at Kumarganj-reclaimed site and at Pundibari site $(\mathrm{r}=-0.57, \mathrm{P}<0.01)$. The grain Se concentration of the 36 genotypes, averaged across years, correlated negatively with juvenile root angle traits at Hisar and Pundibari sites.

\section{Correlations within juvenile root traits in HTP system}

Positive significant relationships were observed within length-related juvenile root traits (CHCRY, CHCRX, CMRX, CMRY, MDR, MWR, TLAR, TLPR, TLLR, RCH) and within juvenile root angle traits (PEA 251, 252, 301, 302, 501, 502, 751, 752, 951, 952, 1001, 1002) $(r=0.42-0.99$; P between $<0.05$ and $<0.001)$. For example, the total length of all roots (TLAR) showed positive relationship $(\mathrm{P}<0.001)$ with total length of primary roots (TLPR) and with maximum depth of root (MDR). Width depth ratio (WDR) and root convex hull $(\mathrm{RCH})$ represent the shape of root system. WDR more strongly associated with maximum width of the root $(\mathrm{MWR})(\mathrm{r}=0.79, \mathrm{P}<0.001)$ than $\mathrm{MDR}(\mathrm{r}=0.02$, $\mathrm{P}=0.9)$. The PEA 252 correlated positively with PEA $752(\mathrm{r}=0.76, \mathrm{P}<0.001)$.

However, some juvenile root length traits, for example, CHCRX, CMRX and MWR also showed positive significant relationships with juvenile root angle traits, PEA 502, PEA 302 and PEA $751(r=0.42, r=0.44, r=0.68$, respectively). Moreover, wider root system associated with the wider/shallower root system (Table S2).

Variance components associated with juvenile root traits and, replications required to detect the differences in juvenile root traits between genotypes

A panel of 36 genotypes showed significant variation in most of the juvenile root traits. Genotype term associated with 13 to $47 \%$ of variations in juvenile root traits under HTP 
system (Table S9; Figure S2). The residual term was associated with 20 to $83 \%$ of variation in juvenile root traits which could be due to variations in seed size and differences between solution containing trays in each growth frames.

The number of replicates required to detect the differences between genotypes for mean root traits under HTP system was measured by constructing the power plot. For example, at $90 \%$ power, to detect the $20 \%$ of difference between genotypes for juvenile root traits for example, MDW, TLPR and $\mathrm{RCH}$, would require 10, 4 and 6 number of replications, respectively (Figure S3).

\section{Discussion}

\section{Relationships between juvenile root traits, grain yield and component traits}

Length related juvenile root traits did not correlate with GYD, BYD, HI and TGW at most of the field sites. This could be due to limited variation in length related juvenile root traits between the Indian wheat genotypes. Our results are consistent with Atkinson et al. (2015), who reported no associations between length-related root traits measured in HTP system and grain yield and component traits of field grown double haploid population of winter wheat. Contrastingly, Xie et al. (2017), reported some positive correlations between juvenile root length traits and GYD of wheat genotypes grown under normal soil field conditions in the UK. The total length of lateral roots and number of lateral roots associated with mature plant height (PHT) of 36 genotypes at most of the field sites except at Kumarganj-sodic and Pundibari (acidic) sites. Our observations are consistent to some extent with Bai et al. (2013) and Atkinson et al. (2015), who reported significant positive relationships between length-related juvenile root traits and mature PHT.

Narrow root angle traits, which potentially represent a steeper root system, correlated with GYD in this study. For example, genotype DBW 71 had narrow root angle (PEA 252 ; 40) and showed greater GYD (3.8 $\mathrm{tha}^{-1}$, averaged across sites and years) than Kharchia $65\left(1.8 \mathrm{t} \mathrm{ha}^{-1}\right)$ which had a wider root angle (PEA 252; 67). Our observations are consistent with McDonald (2010) and Manschadi et al. (2008), who reported that wheat genotypes with narrow juvenile root angles, when grown in soil boxes and gel-filled chamber growth systems, showed greater GYD, than wider root angle genotypes. In addition, narrower juvenile root angles were associated with increased GYD at hostile soils, for example, Kumarganj-reclaimed (saline/reclaimed) and Pundibari (acidic) sites, indicating that narrow root angle may help to increase the grain yield through better absorption of water and nutrients from deeper soil layers under harsh soils. Richard et al. (2015) also reported that narrow root angle could result into the development of compact and deeper root system, which is highly desirable trait in wheat particularly when grown in water deficient soil conditions.

Plants with greater numbers of roots at depth in the soil are likely to make plants more efficient in exploration of sub-surface water and other nutrients under drought and rainfed conditions which may benefit in the improvement of wheat grain production (Manschadi et al. 2010; Richard et al. 2015). 


\section{Relationships between juvenile root traits and grain Zn concentration and the grain ionome}

In the present study, the grain $\mathrm{Zn}$ concentration of 36 genotypes, averaged across years, correlated with TLLR at Pundibari (acidic) site. For example, genotype BH 1146, had highest TLLR $(0.9 \mathrm{~cm})$ and showed highest grain $\mathrm{Zn}$ concentration $\left(33.8 \mathrm{mg} \mathrm{kg}^{-1}\right)$ at Pundibari site, averaged across years, than GW $322\left(22.4 \mathrm{mg} \mathrm{kg}^{-1}\right)$ which had no lateral roots. Increased length of lateral roots can increase the surface area which may help the roots to explore more $\mathrm{Zn}$ in the soil. Rose et al. (2013), also reported that longer lateral roots and longer root hairs increases the acquisition of soil $\mathrm{Zn}$ by roots of rice genotypes.

Wider juvenile root angle traits were positively correlated with grain Ca concentration of the 36 wheat genotypes under hostile soils, whilst length related root traits associated with grain $\mathrm{Mg}$ and $\mathrm{S}$ concentration under hostile and non-hostile soil environments. Lower $\mathrm{Ca}$ concentration in soil is rare in nature, but $\mathrm{Ca}$ deficiency may occur under highly acidic soils and, can affect the plant growth (White and Broadley 2003). Emanuelsson (1984) reported decrease in root length and dry weight of a barley variety under low Ca concentration when grown in pots. Higher $\mathrm{S}$ in soil have shown increase in root number in soybean (Zhao et al. 2008). The wider juvenile root angle traits and lateral root length associated with grain Fe concentration of the 36 wheat genotypes under hostile and nonhostile soil sites. Thomas et al. (2016b) have reported positive association between lateral root density in HTP system and leaf Fe concentration measured in field in oilseed rape genotypes. Therefore, there could be a possibility that wider root system and greater length of lateral roots might increase the efficiency of plants in exploration and acquisition of Fe from the heterogeneous soils. Lateral juvenile root traits were positively correlated with grain $\mathrm{Mn}$ concentration under normal/saline field sites which are in agreement with Liu et al. (2013), who reported positive association between lateral roots and grain $\mathrm{Mn}$ concentration in rice genotypes under field condition. Therefore, developing more dense root system with increase in lateral root length and number could enable the roots to explore more $\mathrm{Mn}$ and $\mathrm{Zn}$ in the mineral rich pockets in the soil (White and Broadley 2009; White and Greenwood 2013). More work is needed to determine the effects of different nutrients, with adequate and limited levels in solution, on juvenile root traits.

The factors responsible for variability in juvenile root traits in HTP system include genotype, environmental factors, $\mathrm{G}^{*} \mathrm{E}$ interaction, experimental design terms (e.g. blocking), and residual terms which include plant-to-plant variation and technical errors. Most of the variation in juvenile root traits was associated with residual term. It is evident that a major source of variation is grain size which affects root and shoot length in wheat (Kakhki et al. 2008; Maydup et al. 2012). Seed size (diameter) was shown to influence root length traits in Brassica napus L. (Thomas et al. 2016a), explaining 44 and 35\% of the variation in total root length and primary root length traits, respectively. Therefore, in this study, we sieved the grain to select a particular range of grain sizes $(3.35-4 \mathrm{~mm}$, diameter) to avoid potential confounding effects of grain size on juvenile root traits. The proportion of residual variation in juvenile root traits could potentially be reduced using a wider panel of genotypes and more replicates. 
Therefore, this HTP system present a huge potential to breeding institutes, for example, the IIWBR, Karnal, India, which has collection of more than 10,000 wheat germplasm lines, could characterise their wider germplasm collections for different root traits before incorporating them into breeding programmes to develop advanced breeding material. Ultimately, the importance of root traits will only be realised when desirable root traits from wild or advanced breeding wheat genotypes are introgressed into modern wheat genotypes and their performance evaluated under different environmental conditions.

\section{Acknowledgements}

We would like to thank all technical staff at all the Indian sites for their help in managing the field experiments and sampling. This work was supported by the Biotechnology and Biological Sciences Research Council [grant number BB/J011827/1], including a studentship to Jaswant S. Khokhar.

\section{References}

Atkinson, J.A., Wingen, L.U., Griffiths, M., Pound, M.P., Gaju, O., Foulkes, M.J. 2015. Phenotyping pipeline reveals major seedling root growth QTL in hexaploid wheat. J. Exp. Bot. 66(8):2283-2292.

Bai, C., Liang, Y., Hawkesford, M.J. 2013. Identification of QTLs associated with seedling root traits and their correlation with plant height in wheat. J. Exp. Bot. 64(6):1745-1753.

Bradley, R.S., Berry, P., Blake, J., Kindred, D., Spink, J., Bingham, I. 2008. "The wheat growth guide", in: The wheat growth guide. (eds) C. Edwards and G.D. Chamberlain. 2 ed.: HGCA).

De Smet, I., White, P.J., Bengough, A.G., Dupuy, L., Parizot, B., Casimiro, I. 2012. Analyzing lateral root development: how to move forward. Plant Cell 24(1):15-20.

Emanuelsson, J. 1984. Root growth and calcium uptake in relation to calcium concentration. Plant Soil 78(3):325-334.

Gewin, V. 2010. Food: An underground revolution. Nature 466(7306):552-553.

Hoad, S.P., Russell, G., Lucas, M.E., Bingham, I.J. 2001. The management of wheat, barley, and oat root systems. Adv. Agron. 74:193-246.

Kakhki, H.T., Kazemi, M., Tavakoli, H. 2008. Analysis of seed size effect on seedling characteristics of different types of wheat (Triticum aestivum L.) cultivars. Asian Journal of Plant Science 7(7):666-671.

Khokhar, J.S., Sareen, S., Tyagi, B.S., Singh, G., Chowdhury, A.K., Dhar, T. 2017. Characterising variation in wheat traits under hostile soil conditions in India. PLoS One 12(6):e0179208.

Khokhar, J.S., Sareen, S., Tyagi, B.S., Singh, G., Wilson, L., King, I.P. 2018. Variation in grain Zn concentration, and the grain ionome, in field-grown Indian wheat. Plos One 13(1):e0192026.

Khush, G.S. 2001. Green revolution: the way forward. Nature Review of Genetics 2(10):815-822.

Liu, Y., Donner, E., Lombi, E., Li, R., Wu, Z., Zhao, F.J. 2013. Assessing the contributions of lateral roots to element uptake in rice using an auxin-related lateral root mutant. Plant Soil 372:125-136.

Lynch, J.P. 2007. Roots of the second green revolution. Aust. J. Bot. 55(5):493-512.

Lynch, J.P. 2013. Steep, cheap and deep: an ideotype to optimize water and $\mathrm{N}$ acquisition by maize root systems. Ann. Bot. 112(2):347-357.

Manschadi, A.M., Christopher, J.T., Hammer, G.L., Devoil, P. 2010. Experimental and modelling studies of drought-adaptive root architectural traits in wheat (Triticum aestivum L.). Plant Biosyst. 144(2):458-462.

Manschadi, A.M., Hammer, G.L., Christopher, J.T., deVoil, P. 2008. Genotypic variation in seedling root architectural traits and implications for drought adaptation in wheat (Triticum aestivum L.). Plant Soil 303:115129. 
Maydup, M.L., Graciano, C., Guiamet, J.J., Tambussi, E.A. 2012. Analysis of early vigour in twenty modern cultivars of bread wheat (Triticum aestivum L.). Crop Pasture Sci. 63(10):987-996.

McDonald, G. 2010. "The effect of root angle on root growth and yield of wheat in the Australian cereal belt", in: Agronomy Conference (15th: 2010: Lincoln, New Zealand. (ed.) H. Dove and R. Culvenor. (New Zealand: NSW, Australia).

Pillinger, C., Paveley, N., Foulkes, M.J., Spink, J. 2005. Explaining variation in the effects of take-all (Gaeumannomyces graminis var. tritici) on nitrogen and water uptake by winter wheat. Plant Pathol. 54(4):491-501.

Pound, M.P., French, A.P., Atkinson, J.A., Wells, D.M., Bennett, M.J., Pridmore, T. 2013. RootNav: navigating images of complex root architectures. Plant Physiol. 162(4):1802-1814.

Richard, C.A., Hickey, L.T., Fletcher, S., Jennings, R., Chenu, K., Christopher, J.T. 2015. High-throughput phenotyping of seminal root traits in wheat. Plant Methods 11:13.

Rose, T.J., Impa, S.M., Rose, M.T., Pariasca-Tanaka, J., Mori, A., Heuer, S. 2013. Enhancing phosphorus and zinc acquisition efficiency in rice: a critical review of root traits and their potential utility in rice breeding. Ann. Bot. 112(2):331-345.

Thomas, C.L., Alcock, T.D., Graham, N.S., Hayden, R., Matterson, S., Wilson, L. 2016a. Root morphology and seed and leaf ionomic traits in a Brassica napus L. diversity panel show wide phenotypic variation and are characteristic of crop habit. BMC Plant Biol. 16(1):214.

Thomas, C.L., Graham, N.S., Hayden, R., Meacham, M.C., Neugebauer, K., Nightingale, M. 2016b. Highthroughput phenotyping (HTP) identifies seedling root traits linked to variation in seed yield and nutrient capture in field-grown oilseed rape (Brassica napus L.). Ann. Bot. 118(4):655-665.

Waines, J.G., Ehdaie, B. 2007. Domestication and crop physiology: roots of green-revolution wheat. Ann. Bot. 100(5):991-998.

White, P.J., Broadley, M.R. 2003. Calcium in plants. Ann. Bot. 92(4):487-511.

White, P.J., Broadley, M.R. 2009. Biofortification of crops with seven mineral elements often lacking in human diets-iron, zinc, copper, calcium, magnesium, selenium and iodine. New Phytol. 182(1):49-84.

White, P.J., Greenwood, D.J. 2013. "Properties and management of cationic elements for crop growth," in Soil Conditions and Plant Growth. Blackwell Publishing Ltd.), pp. 160-194.

Xie, Q., Fernando, K.M.C., Mayes, S., Sparkes, D.L. 2017. Identifying seedling root architectural traits associated with yield and yield components in wheat. Ann. Bot. 119(7):1115-1129.

Zhao, Y.W., Xiao, X., Bi, D.M., Hu, F. 2008. Effects of sulfur fertilization on soybean root and leaf traits, and soil microbial activity. J. Plant Nutr. 31(3):473-483.

\section{Electronic Supplementary Material (ESM)}

Electronic Supplementary Material (ESM) associated with this article can be found at the website of CRC at http://www.akademiai.com/content/120427/

Electronic Supplementary Table S1. Wheat juvenile root traits measured in HTP system

Electronic Supplementary Table S2. Correlation coefficients between wheat juvenile root traits and grain yield, yield components, and grain mineral composition traits. Root data are mean of $\sim 20$ replicates grown in HTP system, yield and yield components data are mean of two replicates each plot, averaged across year and site and mineral composition traits data are means of two replicates per plot per genotype at Karnal, Hisar and Malda, one replicate per plot at Kumarganj-reclaimed and Kumarganj-sodic sites in 2013-2014 and two replicates per plot per genotype at six sites in 2014-2015 
Electronic Supplementary Table S3. Correlation coefficients between wheat juvenile root traits and grain yield, yield components, and grain mineral composition traits of 36 Indian wheat genotypes at Karnal site. Root trait data are the mean of $\sim 20$ replicates, grain yield \& yield components, and mineral composition traits data are the mean of two replicates per plot per genotype over two years at Karnal site

Electronic Supplementary Table S4. Correlation coefficients between wheat juvenile root traits and grain yield, yield components, and grain mineral composition traits of 36 Indian wheat genotypes at Hisar site. Root trait data are the mean of $\sim 20$ replications, grain yield \& yield components, and mineral composition traits data are the mean of two replicates per plot per genotype over two years at Hisar

Electronic Supplementary Table S5. Correlation coefficients between wheat juvenile root traits and grain yield, yield components, and grain mineral composition traits of 36 Indian wheat genotypes at Kumarganj-reclaimed site. Root traits data are the mean of $\sim 20$ replicates, grain yield \& yield components data are the mean of two replicates per plot per genotype over two years at Kumarganj-reclaimed and, mineral composition traits data are the mean of one replicate per plot in 2013-2014 and two replicates per plot per genotype in 2014-2015 at

Kumarganj-reclaimed site

Electronic Supplementary Table S6. Correlation coefficients between wheat juvenile root traits and grain yield, yield components, and grain mineral composition traits of 36 Indian wheat genotypes at Kumarganj-sodic site. Root trait data are the mean of $\sim 20$ replicates, grain yield \& yield components data are the mean of two replicates per plot per genotypes over two years at Kumarganj-sodic and, mineral composition traits data are the mean of one replicate per plot in 2013-2014 and two replicates per plot per genotype in 2014-2015 at

Kumarganj-sodic site

Electronic Supplementary Table S7. Correlation coefficients between wheat juvenile root traits and grain yield, yield components and grain mineral composition traits of 36 Indian wheat genotypes at Malda site. Root trait data are the mean of $\sim 20$ replicates, grain yield \& yield components and, grain mineral composition traits data are the means of two replicates per plot per genotype over two years at Malda site

Electronic Supplementary Table S8. Correlation coefficients between wheat juvenile root traits and grain yield

Electronic Supplementary Table S9. The contribution of genotype, growth frame, replications and residual factors to variation percentage (\%) in juvenile root traits of 36 genotypes grown in HTP system. Data are the means of $\sim 20$ replications

Electronic Supplementary Figure S1. (A) Wheat juvenile root traits (B) Root angles; PEA 301 and PEA 302 represent the paired emergence angle at 30 pixel of root length between out and inner pair of primary roots, respectively $(\mathbf{C})$ Hexagonal area covered by white line showing the convex hull area, smallest area that cover the entire root system

Electronic Supplementary Figure S2. (A) Comparison of wheat genotypes, DBW 16 and BH 1146, with the shortest total length of primary roots (TLPR) and longest TLPR; (B) genotypes KRL 1-4 and BH 1146 with the shortest and longest total length of lateral roots (TLLR); (C) genotypes DPW 621-50 and Kharchia 65 with narrowest and widest emergence angle between the outermost pair of primary roots measured at $25 \%$ length of root (PEA 251), respectively, when grown for ten days in the 'pouch and wick' HTP system (vertical axis length $=30.4 \mathrm{~cm})$

Electronic Supplementary Figure S3. The power of experiment (z-axis, legend (10-90) in percentage) to detect percentage of differences in juvenile root trait means between genotypes (y-axis) as a function of the number of replicates (x-axis) in HTP system 